\title{
Comportamento reológico de néctares mistos de caju, manga e acerola descritos pelo Modelo de Casson
}

\author{
Study of the rheological behaviour of mixed nectars of cashew apple, \\ mango and acerola as described by the Casson Model
}

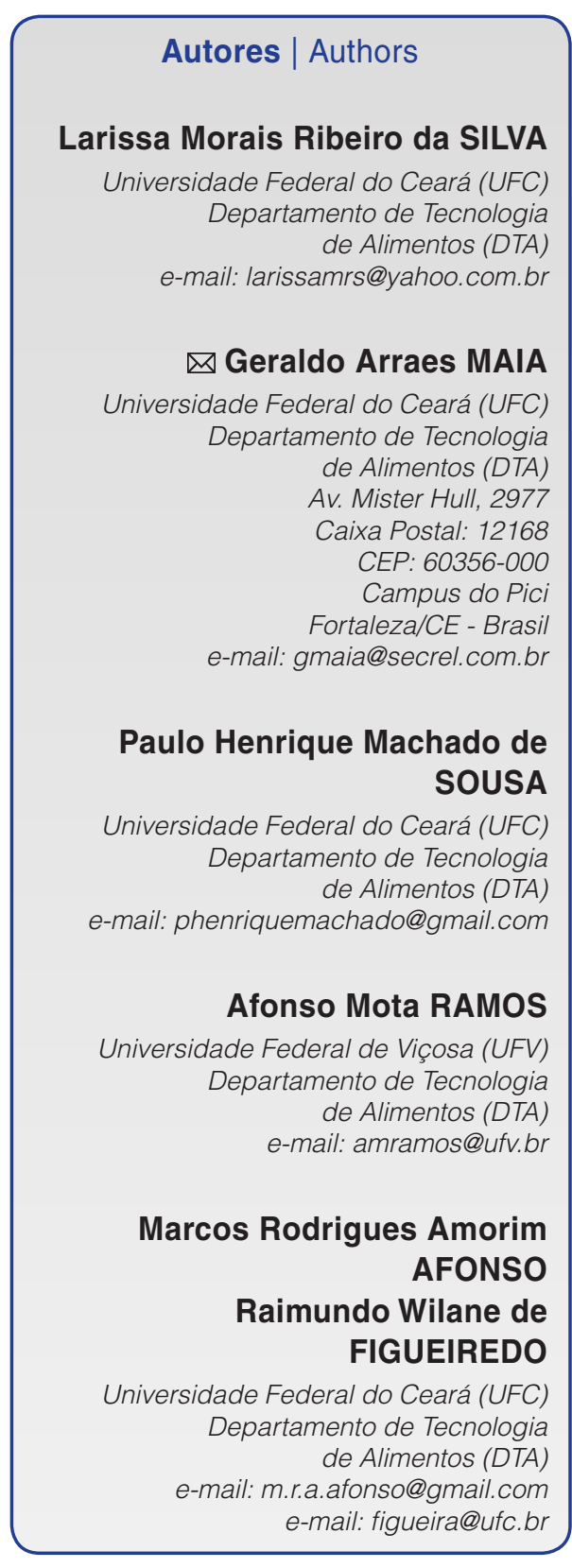

$\triangle$ Autor Correspondente / Corresponding Author

Recebido / Received: 04/11/2010 Aprovado / Approved: 20/06/2012 Publicado / Published: set./2012

\section{Resumo}

A abordagem do comportamento reológico de néctares mistos de frutas tem fornecido importantes informações para o processamento dos mesmos, resultando em benefícios para a indústria e, consequentemente, para o consumidor. Portanto, neste trabalho, se estudou o comportamento reológico de néctares mistos de caju, manga e acerola, nas temperaturas de $8{ }^{\circ} \mathrm{C}$ e $25^{\circ} \mathrm{C}$. As medidas experimentais foram realizadas em reômetro rotacional de cilindros concêntricos e os dados experimentais foram ajustados ao modelo de Casson. A tensão inicial apresentou ajuste ao modelo cúbico especial, para a temperatura de $8^{\circ} \mathrm{C}$. Para a temperatura de $25{ }^{\circ} \mathrm{C}$, observou-se ajuste ao modelo cúbico especial para a viscosidade plástica de Casson. $\mathrm{O}$ aumento nas proporções de polpas de caju, manga e acerola resultou no aumento da viscosidade plástica de Casson e o aumento da quantidade de polpa de caju proporcionou aumento na tensão inicial.

Palavras-chave: Néctar; Frutas tropicais; Análise reológica.

\section{Summary}

The rheological behaviour approach to the study of fruit nectars has provided important information for the processing of these products, resulting in benefits for industry and hence for the consumer. In this paper, the rheological behaviour of mixed beverages based on cashew, mango and acerola fruits was studied at $8{ }^{\circ} \mathrm{C}$ and $25^{\circ} \mathrm{C}$. The experimental measurements were made using a rotating concentric cylinder rheometer and the results adjusted to fit the Casson model. The initial stress fitted the special cubic model at a temperature of $8{ }^{\circ} \mathrm{C}$. At a temperature of $25^{\circ} \mathrm{C}$, it fitted the special cubic model for Casson's plastic viscosity. An increase in the proportions of cashew apple, mango and acerola pulps resulted in an increase in Casson's plastic viscosity and an increase in the amount of cashew apple pulp increased the initial stress.

Key words: Nectar; Tropical fruits; Rheological analysis. 


\section{Introdução}

O consumo de sucos de frutas no Brasil e no mundo encontra-se em plena expansão. Várias frutas encontradas nesses países apresentam composição em aroma e compostos funcionais de grande valor (CAMARGO et al., 2007), como o caju (Anacardium occidentale, L.), a manga (Mangifera indica, L.) e a acerola (Malphigia emarginata, D.C.). O caju é uma fruta tropical nativa do Brasil, seu pedúnculo é utilizado na formulação de diversos produtos como doces e néctares e apresenta elevadores teores de vitamina C (ANDRADE et al., 2008), além de diversas substâncias importantes para a saúde, como carotenóides (ZEPKA e MERCADANTE, 2009) e fenólicos totais (MELO et al., 2008). A manga é uma fruta tropical com alta qualidade em aroma e sabor, apresentando-se como boa fonte de antioxidantes para a dieta humana, com boas quantidades de carotenóides e compostos fenólicos (RIBEIRO et al., 2007).

A acerola tem sido bastante utilizada na formulação de sucos mistos por apresentar elevado conteúdo de fitoquímicos, como os polifenóis, que proporcionam elevada atividade antioxidante (MEZADRI et al., 2008) e a vitamina C (GODOY et al., 2008).

A formulação de néctares mistos de frutas pode ser utilizada com o intuito de melhorar as características nutricionais e sensoriais de determinados sucos por meio da complementação de nutrientes fornecidos por frutas diferentes (LIMA et al., 2008).

Consumidores mais exigentes e com maior poder de compra têm estimulado o desenvolvimento de produtos, o aperfeiçoamento dos processos e as melhorias da qualidade dos produtos agrícolas, resultando na adaptação de sistemas e equipamentos industriais (GRANJEIRO et al., 2007).

Dessa forma, as indústrias de sucos de frutas têm tido a preocupação de melhorar e automatizar a produção desses produtos. Durante o processamento, os sucos de fruta apresentam variações em suas concentrações e temperaturas. Estes são submetidos a operações unitárias, tais como bombeamento, evaporação, secagem e pulverização. Para um correto dimensionamento, operação e controle do processo, o conhecimento dos parâmetros reológicos desses produtos é de fundamental importância (CABRAL et al., 2007).

O comportamento reológico dos néctares mistos de frutas pode ser descrito por diversos modelos empíricos, que relacionam tensão de cisalhamento e taxa de deformação.

O Modelo de Casson (Equação 1) é atualmente bastante utilizado para descrever o comportamento reológico de sucos de fruta por diversos autores (BRANCO e GASPARETO, 2005).
$(\tau)^{0,5}=\mathrm{K}_{\mathrm{oc}}+\kappa_{\mathrm{c}}(\gamma)^{0,5}$

em que: $\tau=$ tensão de cisalhamento $(\mathrm{Pa}) ; \gamma=$ taxa de deformação $\left(\mathrm{s}^{-1}\right) ; \mathrm{K}_{\mathrm{OC}}=$ tensão inicial $(\mathrm{Pa}) ; \mathrm{K}_{\mathrm{c}}=$ viscosidade plástica de Casson (Pa.sn)

O Modelo de Casson foi desenvolvido para descrever um fluido com partículas em suspensão interagindo em um meio newtoniano. A tensão inicial representa uma tensão que deve ser ultrapassada para que o fluido escoe. A viscosidade de Casson representa o grau de resistência do fluido ao escoamento.

Atualmente, no Brasil, são poucos os trabalhos referentes ao estudo do comportamento reológico de néctares mistos de frutas tropicais. Dessa forma, a ausência desses dados poderá levar as indústrias a aplicarem no processamento desses produtos condições semelhantes às utilizadas para néctares de frutas temperadas, o que poderá acarretar erros no desenvolvimento do produto e do processo.

Considerando-se a carência de dados reológicos referentes a néctares mistos de frutas tropicais, este trabalho teve como objetivo analisar o comportamento reológico de néctares mistos de caju, manga e acerola, observando a influência dos teores de cada polpa nas proriedades reológicas do Modelo de Casson.

\section{Material e métodos}

\subsection{Formulações e delineamento experimental}

Foram elaboradas dez formulações com diferentes concentrações de polpas de caju, manga e acerola, fornecidas por uma indústria de Pacajús-CE. As formulações totalizaram $35 \%$ de polpa, a partir de delineamento simplex centroide aumentado para dez formulações (Tabela 1) e as restrições para cada polpa foram: mínimo de $5 \%$ e máximo de $90 \%$. A concentração de sólidos solúveis totais foi fixada em $11^{\circ}$ Brix, por meio de balanço de massa, estando de acordo com o valor estabelecido pela legislação vigente, que estabelece um valor mínimo de $11^{\circ}$ Brix para néctar misto de frutas (BRASIL, 2003). Para o cálculo do balanço de massa, foram obtidos os teores de sólidos solúveis totais ( $\left.{ }^{\circ} B r i x\right)$ por refratometria, em triplicata, utilizando-se refratômetro marca ATAGO, com escala variando de 0 a $90{ }^{\circ}$ Brix, conforme Brasil (2005).

Os néctares mistos foram adicionados de metabissulfito de sódio e benzoato de sódio. As formulações foram submetidas a tratamento térmico de $90{ }^{\circ} \mathrm{C}$ por 1 minuto (pasteurização) em tacho de aço inoxidável, seguindo-se de envase a quente (hot-fill) em garrafas de vidro de $300 \mathrm{~mL}$ e resfriamento em banho de gelo com água clorada até temperatura ambiente (MAIA et al., 2007). As formulações foram mantidas sob 
Tabela 1. Delineamento simplex centroide para dez tratamentos.

\begin{tabular}{|c|c|c|c|c|c|c|}
\hline \multirow{2}{*}{ Formulação } & \multicolumn{3}{|c|}{ Pseudocomponentes } & \multicolumn{3}{|c|}{ Componentes originais } \\
\hline & Caju & Manga & Acerola & Caju & Manga & Acerola \\
\hline 1 & 90 & 5 & 5 & 31,5 & 1,8 & 1,8 \\
\hline 2 & 5 & 90 & 5 & 1,8 & 31,5 & 1,8 \\
\hline 3 & 5 & 5 & 90 & 1,8 & 1,8 & 31,5 \\
\hline 4 & 47,5 & 47,5 & 5 & 16,6 & 16,6 & 1,8 \\
\hline 5 & 47,5 & 5 & 47,5 & 16,6 & 1,8 & 16,6 \\
\hline 6 & 5 & 47,5 & 47,5 & 1,8 & 16,6 & 16,6 \\
\hline 7 & 33,3 & 33,3 & 33,3 & 11,7 & 11,7 & 11,7 \\
\hline 8 & 61,67 & 19,17 & 19,17 & 21,6 & 6,7 & 6,7 \\
\hline 9 & 19,17 & 61,67 & 19,17 & 6,7 & 21,6 & 6,7 \\
\hline 10 & 19,17 & 19,17 & 61,67 & 6,7 & 6,7 & 21,6 \\
\hline
\end{tabular}

congelamento $\left(\sim-18{ }^{\circ} \mathrm{C}\right)$ por aproximadamente duas semanas, até a realização das análises. As formulações foram elaboradas em triplicata, em dias diferentes, resultando em três diferentes lotes. Cada lote foi submetido à análise reológica em duplicata, fornecendo seis diferentes resultados, para cada formulação.

\subsection{Análise reológica}

Levando-se em conta o alto teor de fibras que a polpa de caju apresenta, para a realização das análises reológicas, inicialmente, todas as formulações foram submetidas a um Homogeneizador OMNI MIXER, modelo Omni Macro, da empresa OMNI internacional da cidade Marietta, Georgia, Estados Unidos, probe utilizada $N^{\circ}$ 4264. As amostras foram submetidas a esse homogeneizador a 10000 rpm por um minuto, a fim de diminuir o tamanho das partículas. O comportamento reológico dos néctares mistos de caju, acerola e manga foi determinado por meio de um reômetro rotacional de cilindros concêntricos tipo Searle da Brookfield, modelo $\mathrm{R} / \mathrm{S}$ plus SST 2000. As medidas foram feitas a $8^{\circ} \mathrm{C}$ e $25^{\circ} \mathrm{C}$, ajustadas por meio de um banho termostático marca Brookfield, modelo TC-502, acoplado ao equipamento. Essas temperaturas foram escolhidas por representarem as temperaturas de refrigeração e armazenamento, respectivamente. O equipamento forneceu os dados de tensão de cisalhamento e taxa de deformação por meio do software RHEO V2.8.

As análises reológicas foram obtidas com variação da taxa de deformação de 0 a $500 \mathrm{~s}^{-1}$ (curva ascendente)

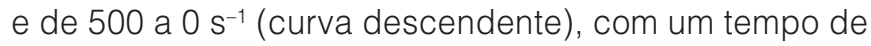
um minuto e leitura de 25 pontos para cada curva. As leituras foram feitas em triplicata e, em cada medida, foi utilizada uma nova amostra. O modelo de Casson foi ajustado aos dados obtidos, por análise de regressão não linear pelo software SAS 9.0.

No ajuste dos dados experimentais, foram testados os modelos linear (Equação 2), quadrático (Equação 3) e cúbico especial (Equation 4), utilizando-se as triplicatas obtidas.

$$
\begin{aligned}
& Y=b_{1}^{\prime} x_{1}^{\prime}+b_{2}^{\prime} x_{2}^{\prime}+b_{3}^{\prime} x_{3}^{\prime} \\
& Y=b_{1}^{\prime} x_{1}^{\prime}+b_{2}^{\prime} x_{2}^{\prime}+b_{3}^{\prime} x_{3}^{\prime}+b_{1}^{\prime} b_{2}^{\prime} x_{1}^{\prime} x_{2}^{\prime}+b_{1}^{\prime} b_{3}^{\prime} x_{1}^{\prime} x_{3}^{\prime}+b_{2}^{\prime} b_{3}^{\prime} x_{2}^{\prime} x_{3}^{\prime} \\
& Y=b_{1}^{\prime} x_{1}^{\prime}+b_{2}^{\prime} x_{2}^{\prime}+b_{3}^{\prime} x_{3}^{\prime}+b_{1}^{\prime} b_{2}^{\prime} x_{1}^{\prime} x_{2}^{\prime}+ \\
& +b_{1}^{\prime} b_{3}^{\prime} x_{1}^{\prime} x_{3}^{\prime}+b_{2}^{\prime} b_{3}^{\prime} x_{2}^{\prime} x_{3}^{\prime}+b_{1}^{\prime} b_{2}^{\prime} b_{3}^{\prime} x_{1}^{\prime} x_{2}^{\prime} x_{3}^{\prime}
\end{aligned}
$$

em que: $Y$ = estimativa da resposta dos parâmetros avaliados, para cada modelo estudado; b'= coeficientes da equação; $x^{\prime}=$ proporção de pseudocomponentes (caju - $x_{1}$; manga $-x_{2}$; acerola $-x_{3}$ ).

A qualidade de ajuste dos modelos foi avaliada com base nos coeficientes de determinação $\left(R^{2}\right)$ próximos à unidade e quadrados médios do erro (QME), próximos a zero.

\section{Resultados e discussões}

Os teores de sólidos solúveis totais obtidos para as polpas de caju, manga e acerola foram de 10,47 \pm 0,06, $14,90 \pm 0,00$ e 5,80 $\pm 0,00$, respectivamente.

Foram observados valores elevados de índice de determinação $\left(R^{2}\right)$ e quadrados médios do erro ( $\left.Q M E\right)$ próximos de zero, indicando bom ajuste do modelo cúbico aos dados obtidos, para as duas temperaturas estudadas (Tabelas 2 e 4).

Para a tensão inicial, $\mathrm{K}_{\mathrm{oc}}$, a $8{ }^{\circ} \mathrm{C}$, foram obtidos valores na faixa de 0,6135 a 1,0412 Pa (Tabela 2). Para a viscosidade plástica de Casson, $K_{c}$, foram obtidos resultados variando na faixa de 0,0506 a 0,0613 Pa.s ${ }^{n}$. Vandressen et al. (2009), analisando os parâmetros reológicos de suco de cenoura, pelo modelo de Casson e à temperatura de $8^{\circ} \mathrm{C}$, mostraram valores para tensão inicial e viscosidade plástica de Casson de 0,864 e 0,0398 , respectivamente. Esses resultados mostram-se próximos dos obtidos neste estudo. 
Tabela 2. Parâmetros reológicos do Modelo de Casson para néctares mistos de caju, acerola e manga para a temperatura de $8{ }^{\circ} \mathrm{C}$, referente ao modelo cúbico especial.

\begin{tabular}{|c|c|c|c|c|c|c|c|}
\hline \multirow{2}{*}{ Néctar } & \multicolumn{3}{|c|}{ Componentes originais } & \multicolumn{4}{|c|}{ Parâmetros } \\
\hline & Caju & Manga & Acerola & $\mathrm{K}_{\mathrm{oc}}(\mathrm{Pa})^{*}$ & $K_{c}\left(P a \cdot s^{n}\right)^{\star}$ & $\mathbf{R}^{2}$ & QME \\
\hline 1 & 31,5 & 1,8 & 1,8 & $0,6512 \pm 0,002$ & $0,0613 \pm 0,000$ & 1,000 & 0,0000 \\
\hline 2 & 1,8 & 31,5 & 1,8 & $0,7465 \pm 0,004$ & $0,0609 \pm 0,000$ & 0,999 & 0,0001 \\
\hline 3 & 1,8 & 1,8 & 31,5 & $0,6135 \pm 0,002$ & $0,0596 \pm 0,000$ & 1,000 & 0,0000 \\
\hline 4 & 16,6 & 16,6 & 1,8 & $1,0412 \pm 0,040$ & $0,0518 \pm 0,002$ & 0,969 & 0,0165 \\
\hline 5 & 16,6 & 1,8 & 16,6 & $0,6823 \pm 0,041$ & $0,0564 \pm 0,002$ & 0,969 & 0,0140 \\
\hline 6 & 1,8 & 16,6 & 16,6 & $0,6810 \pm 0,006$ & $0,0601 \pm 0,000$ & 0,999 & 0,0002 \\
\hline 7 & 11,7 & 11,7 & 11,7 & $0,9139 \pm 0,041$ & $0,0506 \pm 0,002$ & 0,958 & 0,3855 \\
\hline 8 & 21,6 & 6,7 & 6,7 & $0,8938 \pm 0,018$ & $0,0547 \pm 0,001$ & 0,994 & 0,0029 \\
\hline 9 & 6,7 & 21,6 & 6,7 & $0,7394 \pm 0,009$ & $0,0579 \pm 0,001$ & 0,999 & 0,0007 \\
\hline 10 & 6,7 & 6,7 & 21,6 & $0,6312 \pm 0,008$ & $0,0566 \pm 0,000$ & 0,990 & 0,0004 \\
\hline
\end{tabular}

*Valor médio dos parâmetros seguidos dos respectivos desvios padrões.

Os modelos linear, quadrático e cúbico especial obtiveram ajustes significativos $(p \leq 0,05)$ aos dados de tensão inicial $\left(\mathrm{K}_{\mathrm{oc}}\right)$, para a temperatura de $8{ }^{\circ} \mathrm{C}$ (Tabela 3$)$. Por fins ilustrativos, o modelo cúbico especial foi escolhido por fornecer maior índice de determinação $\left(R^{2}\right)$ quando comparado aos demais. A viscosidade plástica de Casson $\left(K_{c}\right)$ não apresentou ajuste significativo a nenhum dos modelos estudados para a temperatura de $8{ }^{\circ} \mathrm{C}$.

Analisando-se os parâmetros reológicos obtidos pelo ajuste dos dados ao Modelo de Casson, a $8{ }^{\circ} \mathrm{C}$, observou-se que a formulação 2 (maior teor de polpa de manga) apresentou maior tensão inicial $\left(K_{o c}\right)$ quando comparada às formulações 1 e 3 (maiores teores de polpas de caju e acerola, respectivamente). Esse comportamento também foi observado para as formulações 4, 5 e 6, e para as formulações 8, 9 e 10, em função da maior proporção de polpas de manga ou caju em suas composições. Dessa forma, o aumento das proporções de polpa de caju e manga resultou em aumento da tensão inicial no escoamento do fluido.
O aumento nas proporções de polpa de caju resultou no aumento da tensão inicial do fluido (Figura 1 e Tabela 4). Quanto maiores os teores de polpas de manga e acerola, menores os valores de tensão inicial $\left(\mathrm{K}_{\mathrm{oc}}\right)$, ou seja, mais facilmente ocorrerá o escoamento do fluido. Haminiuk (2007) avaliou o comportamento reológico de diferentes misturas ternárias de polpas de morango, amora-preta e framboesa, ajustando os dados reológicos ao modelo de Casson, e observou que a polpa de framboesa apresentou um maior efeito na tensão inicial das misturas e que a polpa de morango contribui significativamente para o aumento da viscosidade, na temperatura de $20^{\circ} \mathrm{C}$

O baixo valor de $\mathrm{R}^{2}$ mostra que, apesar da significância estatística para a análise de variância, as equações não são significativas estatisticamente, mas sim indicadoras de tendência.

Para a temperatura de $8{ }^{\circ} \mathrm{C}$, dentre as polpas estudadas, a de caju foi a que mais contribuiu para o aumento da tensão inicial dos néctares, apresentando

Tabela 3. Análise da variância dos modelos linear, quadrático e cúbico especial dos dados de tensão inicial $\left(\mathrm{K}_{\text {oc }}\right)$ e viscosidade plástica de Casson $\left(\mathrm{K}_{c}\right)$, para as temperaturas de 8 e $25^{\circ} \mathrm{C}$.

\begin{tabular}{|c|c|c|c|c|c|c|c|c|c|}
\hline \multirow{3}{*}{$\begin{array}{l}\text { Fonte de variação/ } \\
\text { Modelo }\end{array}$} & \multirow{3}{*}{ G.L } & \multicolumn{8}{|c|}{ Quadrado médio } \\
\hline & & \multicolumn{4}{|c|}{$8^{\circ} \mathrm{C}$} & \multicolumn{4}{|c|}{$25^{\circ} \mathrm{C}$} \\
\hline & & $\mathrm{K}_{\mathrm{oc}}(\mathrm{Pa})$ & $\mathbf{R}^{2}$ & $K_{c}\left(\right.$ Pa. $\left.s^{n}\right)$ & $\mathbf{R}^{2}$ & $\mathrm{~K}_{\mathrm{oc}}(\mathrm{Pa})$ & $\mathbf{R}^{2}$ & $K_{c}\left(P a . s^{n}\right)$ & $\mathbf{R}^{2}$ \\
\hline Linear & 2 & $0,0878^{*}$ & \multirow[t]{2}{*}{0,259} & $0,0048^{\text {ns }}$ & & $0,0859^{*}$ & \multirow[t]{2}{*}{-} & $0,000007^{\text {ns }}$ & \multirow[t]{2}{*}{-} \\
\hline F.A & 7 & $0,0189^{n s}$ & & $0,0114^{\text {ns }}$ & & $0,0525^{*}$ & & $0,000047^{*}$ & \\
\hline Quadrático & 5 & $0,0534^{*}$ & \multirow[t]{2}{*}{0,394} & $0,0125^{\mathrm{ns}}$ & & $0,0938^{*}$ & \multirow[t]{2}{*}{-} & $0,000061^{*}$ & \multirow[t]{2}{*}{0,519} \\
\hline F.A & 4 & $0,0103^{\text {ns }}$ & & $0,0068^{\text {ns }}$ & & $0,0176^{*}$ & & $0,000008^{\text {ns }}$ & \\
\hline Cúbico especial & 6 & $0,0455^{\star}$ & \multirow[t]{2}{*}{0,402} & $0,0136^{n s}$ & & $0,0782^{*}$ & \multirow[t]{2}{*}{-} & $0,000052^{*}$ & \multirow[t]{2}{*}{0,395} \\
\hline F.A & 3 & $0,0118^{\text {ns }}$ & & $0,0027^{\mathrm{ns}}$ & & $0,0235^{\star}$ & & $0,000009^{n s}$ & \\
\hline$F^{\star *}$ & & \multicolumn{2}{|c|}{$4,7114^{*}$} & \multicolumn{2}{|c|}{$0,4615^{\mathrm{ns}}$} & \multicolumn{2}{|l|}{$5,0452^{*}$} & \multicolumn{2}{|l|}{$0,3111^{\mathrm{ns}}$} \\
\hline F.A & & \multicolumn{2}{|c|}{$1.0248^{\mathrm{ns}}$} & \multicolumn{2}{|c|}{$1.1457^{\mathrm{ns}}$} & $11,326^{*}$ & & \multicolumn{2}{|l|}{$3.7125^{\star}$} \\
\hline
\end{tabular}

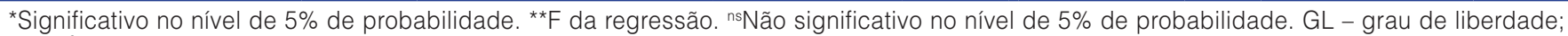
F.A - falta de ajuste. 
Tabela 4. Efeitos do modelo cúbico especial nos parâmetros reológicos do Modelo de Casson.

\begin{tabular}{|c|c|c|c|c|}
\hline \multirow{3}{*}{ Variáveis** } & \multicolumn{4}{|c|}{ Efeito } \\
\hline & \multicolumn{2}{|c|}{$8^{\circ} \mathrm{C}$} & \multicolumn{2}{|c|}{$25^{\circ} \mathrm{C}$} \\
\hline & $\mathrm{K}_{\mathrm{oc}}(\mathrm{Pa})$ & $K_{c}\left(P a \cdot s^{n}\right)$ & $\mathrm{K}_{\mathrm{oc}}(\mathrm{Pa})$ & $K_{c}\left(P a \cdot s^{n}\right)$ \\
\hline$b_{1}$ & $10,7983^{*}$ & $1,6107^{\text {ns }}$ & $14,3372^{*}$ & $31,6515^{\star}$ \\
\hline$b_{2}$ & $9,2750^{*}$ & $1,1835^{\text {ns }}$ & $15,2380^{*}$ & $31,7556^{*}$ \\
\hline$b_{3}$ & $8,7516^{*}$ & $1,2319^{n s}$ & $12,7865^{\star}$ & $30,7158^{*}$ \\
\hline$b_{1} b_{2}$ & $0,9578^{\text {ns }}$ & $0,0647^{\text {ns }}$ & $5,7792^{\star}$ & $-3,8036^{\star}$ \\
\hline$b_{1} b_{3}$ & $0,2505^{n s}$ & $-0,0292^{\text {ns }}$ & $0,7892^{\text {ns }}$ & $-1,5264^{\text {ns }}$ \\
\hline$b_{2} b_{3}$ & $-1,8636^{\star}$ & $2,6406^{\star}$ & $-0,2737^{\mathrm{ns}}$ & $-0,0104^{\mathrm{ns}}$ \\
\hline$b_{1} b_{2} b_{3}$ & $0,5702^{\text {ns }}$ & $-1,4436^{\mathrm{ns}}$ & $0,1759^{\text {ns }}$ & $-0,7741^{\text {ns }}$ \\
\hline
\end{tabular}

${ }^{*}$ Significativo no nível de $5 \%$ de probabilidade. ${ }^{*}$ Variáveis relativas às polpas de caju $\left(b_{1}\right)$, manga $\left(b_{2}\right)$ e acerola $\left(b_{3}\right)$. nsNão significativo no nível de $5 \%$ de probabilidade.

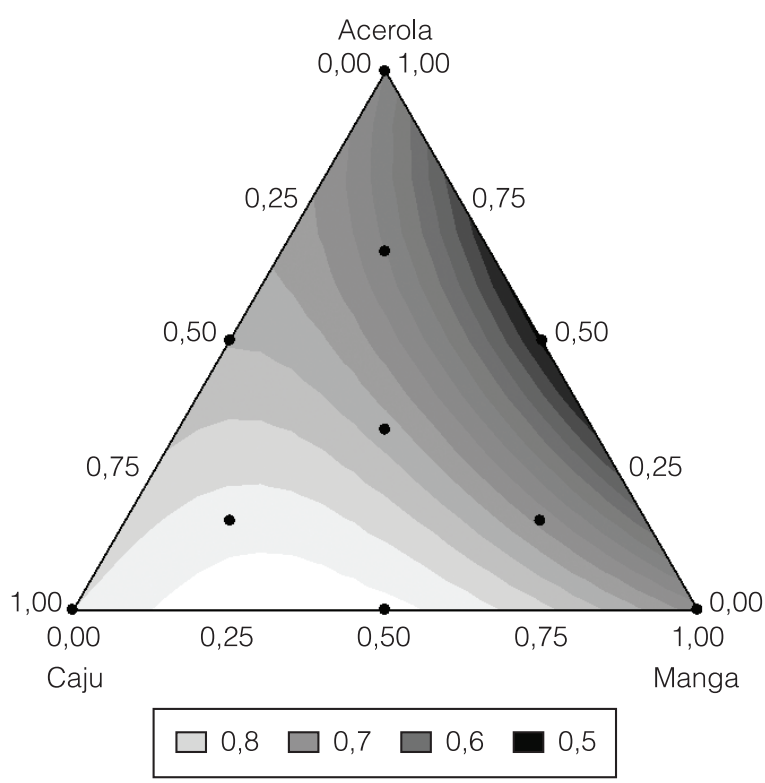

Figura 1. Diagrama ternário da superfície de resposta do modelo cúbico especial relativo à tensão inicial $\left(K_{o c}\right)$ a $8{ }^{\circ} \mathrm{C}$. Caju $-x_{1}$; Manga $-x_{2}$; Acerola $-x_{3} \cdot R^{2}=0,402$

maior valor de coeficiente entre os demais observados, seguido das polpas de manga e acerola, respectivamente. A interação entre as polpas de manga e acerola contribuiu para a diminuição da tensão inicial, a $8^{\circ} \mathrm{C}$, e a interação entre as três polpas contribuiu para o aumento da mesma.

Para a tensão inicial, a $25{ }^{\circ} \mathrm{C}$, foram obtidos valores na faixa de 0,6026 a 0,8846 Pa (Tabela 5). Para a viscosidade plástica de Casson, $\mathrm{K}_{c}$, foram obtidos resultados variando na faixa de 0,0702 a 0,0827 Pa.sn.

Vandressen et al. (2009), analisando os parâmetros reológicos de suco de cenoura, por meio do modelo de Casson a $25^{\circ} \mathrm{C}$, obtiveram valores para tensão inicial e viscosidade plástica de Casson de 0,740 e 0,0328 Pa. $\mathrm{S}^{n}$, respectivamente. Esses resultados mostram-se próximos dos obtidos neste estudo para a tensão inicial.

Para a temperatura de $25^{\circ} \mathrm{C}$, os dados de viscosidade plástica de Casson $\left(K_{c}\right)$ obtiveram ajustes significativos $(p \leq 0,05)$, para os modelos quadrático e cúbico especial. Para a representação, o modelo cúbico especial foi escolhido por fornecer maior índice de determinação $\left(R^{2}\right)$ quando comparado aos demais. $A$ tensão inicial $\left(\mathrm{K}_{o c}\right)$ não apresentou ajuste significativo a nenhum dos modelos estudados.

Pode ser verificado que o aumento nas proporções de polpas de caju, manga e acerola (vértices do diagrama) acarreta o aumento da viscosidade plástica de Casson (Figura 2). Dessa forma, as formulações 1, 2 e 3 tendem a ter maior valor de viscosidade plástica de Casson. Quando são aumentadas as proporções de polpas de caju e manga, em quantidades similares na formulação, observa-se uma redução da viscosidade plástica de Casson $\left(\mathrm{K}_{\mathrm{c}}\right)$.

A polpa de manga foi a que mais contribuiu para o aumento da viscosidade plástica de Casson $\left(K_{c}\right)$ dos néctares, apresentando maior valor de coeficiente entre os demais observados, seguida das polpas de caju e acerola.

A interação entre as polpas de caju e manga contribuiu para o aumento na viscosidade plástica de Casson $\left(K_{c}\right)$, enquanto a interação entre as polpas de caju e acerola resultou em leve decréscimo.

Com o aumento das proporções de polpas de manga e caju, as formulações apresentaram uma tendência a maiores valores de tensão inicial, sendo tal comportamento contrário ao observado com o aumento das proporções de polpa de acerola.

Branco e Gasparetto (2005) avaliaram o comportamento reológico de misturas de polpa de manga e sucos de laranja e cenoura, e observaram, para todas as formulações estudadas, que a tensão inicial de Casson aumentou com o aumento da fração de manga e diminuiu com o aumento das frações de laranja e cenoura.

Para a viscosidade plástica de Casson $\left(\mathrm{K}_{\mathrm{c}}\right)$, de um modo geral, pode-se afirmar que as formulações 
Comportamento reológico de néctares mistos de caju, manga e acerola descritos pelo Modelo de Casson SILVA, L. M. R. et al.

Tabela 5. Parâmetros reológicos do Modelo de Casson para néctares mistos de caju, acerola e manga para a temperatura de $25^{\circ} \mathrm{C}$

\begin{tabular}{|c|c|c|c|c|c|c|c|}
\hline \multirow{2}{*}{ Néctar } & \multicolumn{3}{|c|}{ Componentes originais } & \multicolumn{4}{|c|}{ Parâmetros } \\
\hline & Caju & Manga & Acerola & $\mathrm{K}_{\mathrm{oc}}(\mathrm{Pa})^{*}$ & $\mathrm{~K}_{\mathrm{c}}\left(\mathrm{Pa} \cdot \mathrm{s}^{n}\right)^{*}$ & $\mathbf{R}^{2}$ & QME \\
\hline 1 & 31,5 & 1,8 & 1,8 & $0,7778 \pm 0,005$ & $0,0766 \pm 0,000$ & 0,999 & 0,000 \\
\hline 2 & 1,8 & 31,5 & 1,8 & $0,7096 \pm 0,061$ & $0,0702 \pm 0,003$ & 0,957 & 0,039 \\
\hline 3 & 1,8 & 1,8 & 31,5 & $0,6723 \pm 0,012$ & $0,0722 \pm 0,000$ & 0,998 & 0,002 \\
\hline 4 & 16,6 & 16,6 & 1,8 & $0,7552 \pm 0,102$ & $0,0827 \pm 0,006$ & 0,916 & 0,147 \\
\hline 5 & 16,6 & 1,8 & 16,6 & $0,7461 \pm 0,056$ & $0,0710 \pm 0,003$ & 0,965 & 0,034 \\
\hline 6 & 1,8 & 16,6 & 16,6 & $0,7268 \pm 0,027$ & $0,0737 \pm 0,001$ & 0,993 & 0,007 \\
\hline 7 & 11,7 & 11,7 & 11,7 & $0,7349 \pm 0,007$ & $0,0751 \pm 0,000$ & 0,999 & 0,000 \\
\hline 8 & 21,6 & 6,7 & 6,7 & $0,8846 \pm 0,036$ & $0,0723 \pm 0,002$ & 0,985 & 0,017 \\
\hline 9 & 6,7 & 21,6 & 6,7 & $0,6470 \pm 0,010$ & $0,0713 \pm 0,000$ & 0,999 & 0,001 \\
\hline 10 & 6,7 & 6,7 & 21,6 & $0,6026 \pm 0,013$ & $0,0762 \pm 0,000$ & 0,998 & 0,002 \\
\hline
\end{tabular}

*Valor médio dos parâmetros seguidos dos respectivos desvios padrões.

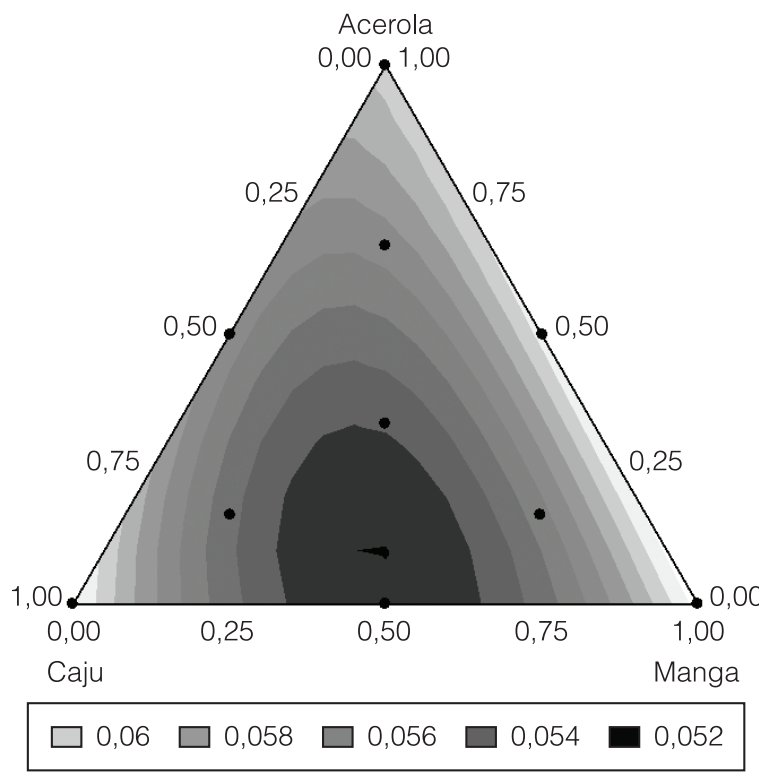

Figura 2. Diagrama ternário da superfície de resposta do modelo cúbico especial relativo à viscosidade plástica de Casson $\left(\mathrm{K}_{\mathrm{c}}\right)$ a $25^{\circ} \mathrm{C}$. Caju $-\mathrm{x}_{1}$; Manga $-\mathrm{x}_{2}$; Acerola $-\mathrm{x}_{3}$. $\mathrm{R}^{2}=0,532$.

com maiores teores de polpas de caju e/ou manga apresentaram tendência a resultados maiores.

\section{Conclusões}

O Modelo de Casson forneceu bons ajustes estatísticos aos dados reológicos para todas as formulações estudadas. O aumento nas proporções de polpas de caju, manga e acerola resultou no aumento da viscosidade plástica de Casson $\left(\mathrm{K}_{\mathrm{c}}\right)$, a $25^{\circ} \mathrm{C}$, e o aumento da polpa de caju e manga proporcionou aumento na tensão inicial $\left(\mathrm{K}_{\mathrm{oc}}\right)$, a $8^{\circ} \mathrm{C}$.

\section{Agradecimentos}

À CAPES e ao CNPq.

\section{Referências}

ANDRADE, A. P. S.; OLIVEIRA, V. H.; INNECCO, R.; SILVA, E. O. Qualidade de cajus-de-mesa obtidos nos sistemas de produção integrada e convencional. Revista Brasileira de Fruticultura, Jaboticabal, v. 30, n. 1, p. 176-179, 2008. http:// dx.doi.org/10.1590/S0100-29452008000100032

BRANCO, I. G.; GASPARETTO, C. A. Comportamento reológico de misturas de polpa de manga utilizando modelo de Casson. Brazilian Journal of Food Technology, Campinas, v. 8, n. 2, p. 183-189, 2005

BRASIL. Ministério da Saúde. Agência Nacional de Vigilância Sanitária. Métodos Físico-Químicos para Análise de Alimentos. Brasília: Ministério da Saúde, 2005. 1018 p.

BRASIL. Ministério da Agricultura, Pecuária e Abastecimento. Instrução Normativa $n^{\circ} 12$ de 4 de set. 2003. Padrões de Identidade para Sucos e Néctares. Diário Oficial da República Federativa do Brasil, Brasília, DF, n. 174, 09 set. 2003.

CABRAL, R. A. F.; ORREGO-ALZATE, C. E.; GABAS, A. L.; TELIS-ROMERO, J. Rheologycal and termophysical properties of blackberry juice. Ciência e Tecnologia de Alimentos, Campinas, v. 27, n. 3, p. 589-596, 2007. http://dx.doi. org/10.1590/S0101-20612007000300025

CAMARGO, G. A.; CONSOLI, L.; LELLIS, I. C. S.; MIELI, J. SASSAKI, E. K. Bebidas naturais de frutas: perspectivas de mercado, componentes funcionais e nutricionais. Bio Engenharia, Campinas, v.1, n. 2, p. 179-205, 2007.

GODOY, R. C. B.; MATOS, E. L. S.; AMORIM, T. S.; SOUZA NETO, M. A.; RITZINGER, R.; WASZCZYNSKY, J. Avaliação de genótipos e variedades de acerola para consumo in natura e para elaboração de doces. Boletim do Centro de Pesquisa de Processamento de Alimentos, Curitiba, v. 26, n. 2, p. 197-204, 2008.

GRANJEIRO, A. A.; QUEIROZ, A. J. M.; FIGUEIRÊDO, F. M. F.; MATA, R. M. E. R. M. C. Viscosidades de polpas concentradas 
Comportamento reológico de néctares mistos de caju, manga e acerola descritos pelo Modelo de Casson SILVA, L. M. R. et al.

de figo da índia. Revista Brasileira de Agrociência, Pelotas, v. 13, n. 2, p.219-224, 2007.

HAMINIUK, C. W. I. Estudo do Comportamento Reológico e Colorimétrico de Misturas Ternárias e Sistemas Pécticos de Polpas de Morango, Amora-preta e Framboesa. 2007. $147 \mathrm{f}$. Tese (Doutorado)-Universidade Federal do Paraná, Curitiba, 2007.

LIMA, A. S.; MAIA, G. A.; SOUSA, P. H. M.; SILVA, F. G. V.; FIGUEIREDO, E. A. T. Desenvolvimento de bebida mista a base de água de coco e suco de acerola. Ciência e Tecnologia de Alimentos, Campinas, v. 28, n. 3, p. 683-690, 2008. http://dx.doi. org/10.1590/S0101-20612008000300026

MAIA, G. A.; SOUSA, P. H. M.; LIMA, A. S. L. Processamento de Frutas Tropicais. Fortaleza: Edições UFC, 2007. 320 p.

MELO, E. A.; MACIEL, M. I. S.; LIMA, V. L. A. G.; ARAÚJO, C. R. Teor de fenólicos totais e capacidade antioxidante de polpas congeladas de frutas. Alimentos e Nutrição, Araraquara, v. 19, n. 1, p. 67-72, 2008.

MEZADRI, T.; VILLAÑO, D.; FERNÁNDEZ-PACHÓN, M. S.; GARCÍAPARILLA, M. C; TRONCOSO, A. M. Antioxidants compounds and antioxidant activity in acerola (Malpighia Emarginata, D.C.) fruits and derivatives. Journal of Food Composition and Analysis, Oxford, v. 21, n. 4, p. 282- 290, 2008. http://dx.doi.org/10.1016/j. jfca.2008.02.002

RIBEIRO, S. M. R.; QUEIROZ, J. H.; QUEIROZ, M. E. L. R.; CAMPOS, F. M.; SANT'ANA, H. M. P. Antioxidant in mango (Mangifera indica L.) pulp. Plant Foods for Human Nutrition, New York, v. 62, n. 1, p. 13-17, 2007. http://dx.doi.org/10.1007/ s11130-006-0035-3

VANDRESSEN, S.; QUADRI, M. G. N.; SOUZA, J. A. R.; HOTZA, D. Temperature effect on the rheological behavior of carrot juices. Journal of Food Engeneering, Essex, v. 92, n. 3, p. 269-274, 2009. http://dx.doi.org/10.1016/j. jfoodeng.2008.11.010

ZEPKA, L. Q.; MERCADANTE, A. Z. Degradation compounds of carotenoids formed during heating of a simulated cashew apple juice. Food Chemistry, Oxford, v. 117, n. 1, p. 28-34, 2009. http://dx.doi.org/10.1016/j.foodchem.2009.03.071 